\title{
Gene expression profile of the intima and media of experimentally induced cerebral aneurysms in rats by laser-microdissection and microarray techniques
}

\author{
TOMOHIRO AOKI, HIROHARU KATAOKA, RYOTA ISHIBASHI, \\ KAZUHIKO NOZAKI and NOBUO HASHIMOTO
}

\author{
Department of Neurosurgery, Kyoto University Graduate School of Medicine, \\ 54 Kawaharacho, Shogoin, Sakyo-ku, Kyoto 606-8507, Japan
}

Received August 21, 2007; Accepted November 19, 2007

DOI: 10.3892/ijmm_00000061

\begin{abstract}
Cerebral aneurysm is a common disease with a high prevalence and can cause a catastrophic subarachnoid hemorrhage. To elucidate the molecular mechanism of the formation and progression of cerebral aneurysms, gene expression profiling was performed in experimentally induced rat cerebral aneurysms. The intima and media of cerebral arterial walls in rats with or without aneurysm induction were dissected respectively by a laser-microdissection technique. Changes in gene expression in the intima and media of aneurysmal walls were analyzed using Agilent Rat Oligo Microarrays, followed by a specific pathway analysis using GeneSpring software. Of the 41,012 genes examined, 633 were differentially expressed between a normal cerebral artery and a cerebral aneurysm in the intima, with 395 showing increased expression and 238 showing decreased expression. In the media, 1344 were differentially expressed, with 928 showing increased expression and 416 showing decreased expression. Specific pathway analysis revealed that increased gene expression was associated with proteinase, reactive oxygen species, growth factor, chemokine, complement, adhesion molecule and apoptosis in both the intima and the media of aneurysmal walls. Some genes showed an opposite expression pattern between the intima and the media indicating a different role between endothelial cells and vascular smooth muscle cells in cerebral aneurysm formation and progression. These data suggest that cerebral aneurysmal formation and progression are closely related to vascular inflammation, degeneration of extracellular matrix and apoptosis.
\end{abstract}

Correspondence to: Dr Hiroharu Kataoka, Department of Neurosurgery, Kyoto University Graduate School of Medicine, 54 Kawaharacho, Shogoin, Sakyo-ku, Kyoto 606-8507, Japan

E-mail: kataoka@kuhp.kyoto-u.ac.jp

Key words: animal model, cerebral aneurysm, gene expression profile, inflammation, laser-microdissection, T7 RNA amplification

\section{Introduction}

Cerebral aneurysm (CA) is a common disease with a high prevalence ranging from 1 to 5 percent in large autopsy studies and CA can cause a catastrophic subarachnoid hemorrhage, one of the most severe forms of stroke (1). Despite the recent advances in the treatment of CAs, the outcome of patients with aneurysmal subarachnoid hemorrhage has not significantly improved. Given the devastating consequences of aneurysmal subarachnoid hemorrhage, development of a novel medical treatment to prevent the growth and rupture of CAs is essential. The pathophysiology of human CA is complex and involves multiple factors, such as the accumulation of inflammatory cells (2), excessive degeneration of the extracellular matrix (ECM) in aneurysmal walls (3) and apoptotic cell death in medial smooth muscle cells (SMCs) $(4,5)$. Progress in understanding the mechanisms underlying the initiation and progression of CA has been hampered by its multi-factorial nature. As two cell types, endothelial cells (ECs) and SMCs, exist in normal cerebral vascular walls, it is important to elucidate the role of each cell type in CA formation and progression.

We developed an animal model of experimentally induced CAs in rats (6), mice (7) and monkeys (8). In this model, CAs were spontaneously induced by hemodynamic stress and systemic hypertension (6). The histopathological features are closely similar to human CAs (9). In an attempt to elucidate the molecular events occurring in ECs and SMCs, respectively, during the process of $\mathrm{CA}$ formation and progression, we dissected the intima and media from rat CA samples by a laser-captured microdissection technique and performed a gene array analysis in each sample. This is the first study of DNA microarray analysis for CAs.

\section{Materials and methods}

Induction of experimentally induced cerebral aneurysms. Cerebral aneurysms were induced as previously described by Nagata et al (10). After the induction of pentobarbital anesthesia $(50 \mathrm{mg} / \mathrm{kg}$ i.p.), the left common carotid artery and posterior branches of the bilateral renal arteries were ligated at the same time with 10-0 nylon in 7 week-old male Sprague-Dawley rats (Oriental Bioservice, Osaka, Japan). 
Animals were fed special food containing $8 \%$ sodium chloride and $0.12 \%$ B-aminopropionitrile (BAPN) (Tokyo Chemical, Tokyo, Japan), an inhibitor of lysyl oxidase that catalyzes the cross-linking of collagen and elastin. Animal care and experiments complied with the Japanese community standards on the care and use of laboratory animals.

Tissue preparation and laser-captured microdissection. One month after aneurysm induction, rats were deeply anesthetized. An age-matched male Sprague-Dawley rat served as a control. The anterior cerebral artery/olfactory artery (ACA/ OA) bifurcation was stripped in RNAlater RNA Stabilization Reagent (Qiagen, Hilden, Germany) under a surgical microscope, embedded in OCT compound (Sakura, Tokyo, Japan) and rapidly frozen in liquid nitrogen. Sections $(5 \mu \mathrm{m})$ were cut and mounted on foiled slides (Leica Microsystems Japan, Tokyo, Japan). After fixation, slides were incubated with Toluidine blue ( $\mathrm{pH}$ 7.0) for $30 \mathrm{sec}$ (Wako, Tokyo, Japan) and air-dried. From the tissue on the slides, the intimal portion of the cerebral aneurysms was initially dissected and then the medial portion was dissected by a laser-captured microdissection technique (Leica Microsystems Japan) (Fig. 1).

RNA isolation, amplification and reverse transcription. Total RNA from the dissected tissue was isolated using the RNeasy Micro Kit (Qiagen) with 20 ng of carrier RNA, followed by the T7 RNA polymerase RNA amplification method by using the TargetAmp Aminoallyl-aRNA Amplification Kit (AR Brown, Tokyo, Japan) according to the manufacturer's directions. Briefly, the first cDNA synthesized by SuperScript reverse transcriptase (Invitrogen, Carlsbad, CA) and T7-oligo (dT) primer was subjected to in vitro transcription. Amplified RNA was again reverse-transcribed and amplified by in vitro transcription by using aminoallyl-UTP. Finally, aminoallylamplified RNA was purified by the RNeasy Mini Kit (Qiagen). The amount of amplified RNA was quantified by a spectrophotometer (NanoDrop, Wilmington, DE). The quality of the amplified RNA was confirmed by gel electrophoresis and by the absorbance ratio $(\mathrm{A} 260 / \mathrm{A} 280$ ratio $=1.9-2.1)$.

DNA microarray. Aminoallyl-amplified RNA was labeled with $\mathrm{Cy} 3$ (for the control rat) or Cy5 (for a rat with aneurysm induction) and reverse transcribed into cDNA by $\mathrm{Cy} 3$ or $\mathrm{Cy} 5$ monoreactive dye (AR Brown). Cy3- or Cy5-labeled cDNA was purified using the RNeasy Mini Kit, fragmented, and applied to the Rat Oligo Microarray (Agilent, Santa Clara, CA) for $17 \mathrm{~h}$ at $65^{\circ} \mathrm{C}$. After washing, microarray plates were scanned by Agilent Technologies Microarray Scanner (Agilent). The ratio of $\mathrm{Cy} 5 / \mathrm{Cy} 3$ was measured on the same plate and genes with a $\mathrm{Cy} 5 / \mathrm{Cy} 3$ ratio $>1.5$ were considered significant. Data were analyzed by the use of GeneSpring software ver. 7.3.1 (Agilent) for a specific pathway analysis. The selected pathways were proteinase, reactive oxygen species (ROS), growth factor, chemokine, complement, adhesion molecule and apoptosis.

\section{Results}

Gene expression profile in the intima of aneurysmal walls in rats (Table I). Of the 41,012 genes examined, 633 genes

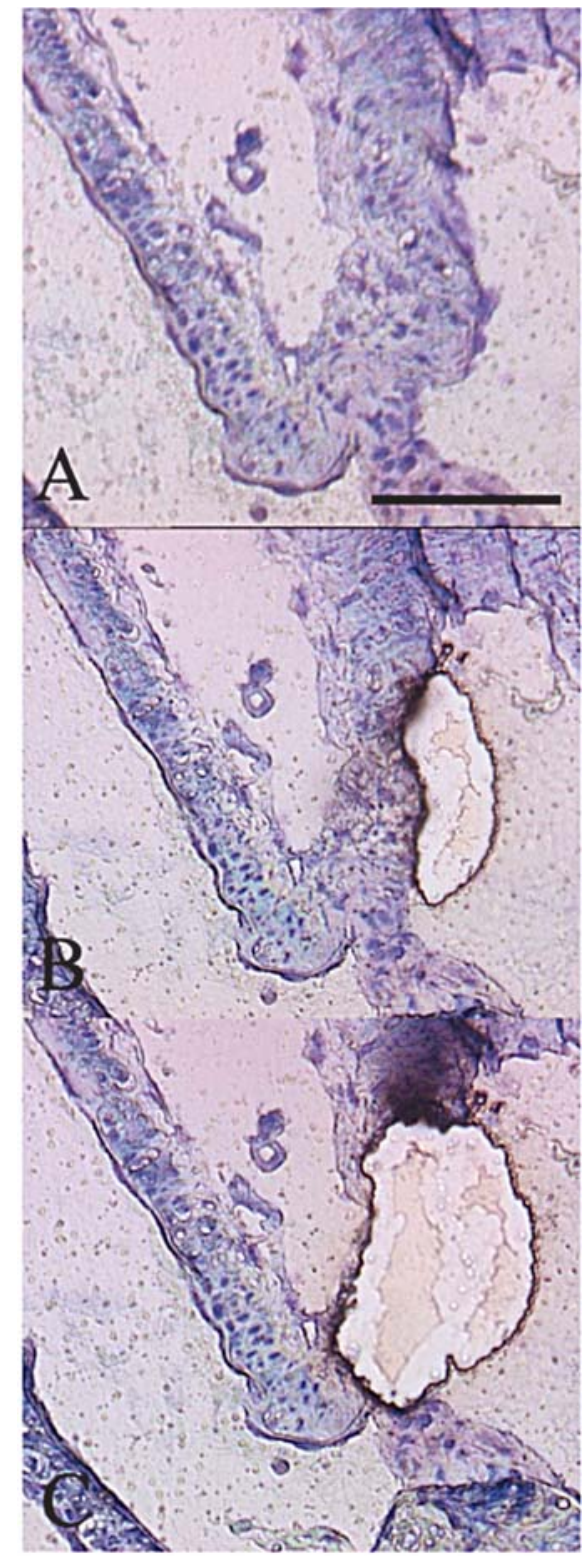

Figure 1. A representative photograph of experimentally induced rat cerebral aneurysms before and after laser-microdissection. (A) An ACA/OA bifurcation one month after aneurysm induction before laser-microdissection. (B) The same section after dissection of the intimal portion, and (C) the same section after dissection of the medial portion. Bar, $100 \mu \mathrm{m}$. The intimal and medial portions of the aneurysm were successfully dissected by the laser-microdissection technique.

$(1.54 \%)$ were differentially expressed between a normal cerebral artery and a cerebral aneurysm in the intima, with 395 genes showing increased expression and 238 genes showing decreased expression. Certain proteinase genes were upregulately expressed in the intima, including matrix metalloproteinase (MMP)-2 and -3 , and cathepsin Z. In reactive oxygen species (ROS)-related genes, both superoxideproducing genes such as NADPH oxidase and superoxideeliminating genes such as superoxide dismutase-2 (SOD-2) were upregulated in the intima. Various growth factors for family members of fibroblast growth factor (FGF), transforming growth factor (TGF) and bone morphogenic protein (BMP) were upregulated in the intima. Important chemokines including monocyte chemoattracting protein-1 
Table I. List of genes whose expression changed in the intima.

\begin{tabular}{ll}
\hline Cascade & \multicolumn{1}{c}{ Gene [Gene accession no. (NM_)] } \\
\hline Proteinase & Serine (or cysteine) peptidase inhibitor, clade E, member 1 (Serpine1) [12620] \\
& Cathepsin Z [183330] \\
& Proteasome (prosome, macropain) subunit, $\alpha$ type 1 (Psma1) [17278] \\
& Matrix metallopeptidase 2 (Mmp2) [31054] \\
& Matrix metallopeptidase 3 (Mmp3) [133523] \\
& Serine (or cysteine) peptidase inhibitor, clade G, member 1 (Serping1) [199093] \\
& Peroxiredoxin 2 (Prdx2) [17169] \\
& Aldehyde dehydrogenase family 3, subfamily A2 (Aldh3a2) [31731] \\
& Catalase [12520] \\
ROS & Superoxide dismutase 2, mitochondrial (Sod2) [17051] \\
& Transaldolase 1 [31811] \\
& Epoxide hydrolase 2, cytoplasmic (Ephx2) [22936] \\
& Peptidylglycine $\alpha$-amidating monooxygenase (Pam) [13000] \\
& Flavin containing monooxygenase 2 (Fmo2) [144737] \\
& Peroxiredoxin 6 (Prdx6) [53576] \\
& NADPH oxidase 4 (Nox4) [53524]
\end{tabular}

Growth factor

Fibroblast growth factor 20 (Fgf20) [23961]

Hepatocyte growth factor activator (Hgfac) [53320]

Neurotrophic tyrosine kinase, receptor, type 1 (Ntrk1) [21589]

Myotrophin [24374]

Transforming growth factor $ß$ regulated gene 4 (Tbrg4) [1012154]

Vascular endothelial growth factor B (Vegfb) [53549]

Bone morphogenetic protein 4 (Bmp4) [12827]

Vascular endothelial growth factor A (Vegfa) [31836]

Transforming growth factor, $\beta$ receptor III (Tgfbr3) [17256]

Interleukin 4 [201270]

Fibroblast growth factor 5 (Fgf5) [22211]

Fibroblast growth factor 12 (Fgf12) [130814]

Transforming growth factor, $\beta$ receptor II (Tgfbr2) [31132]

Latent transforming growth factor $\beta$ binding protein 2 (Ltbp2) [21586]

Bone morphogenetic protein 6 (Bmp6) [13107]

Growth factor receptor bound protein 2 (Grb2) [30846]

Neuropilin 1 [145098]

Fibroblast growth factor 3 (Fgf3) [130817]

Jagged 1 [19147]

Bone morphogenetic protein 15 (Bmp15) [21670]

Fibroblast growth factor binding protein 1 (Fgfbp1) [22603]

Fibroblast growth factor 10 (Fgf10) [12951]

Early growth response 3 (Egr3) [17086]

Fibroblast growth factor 4 (Fgf4) [53809]

Chemokine

\author{
Chemokine (C-X-C motif) ligand 7 (Cxcl7) [153721] \\ Chemokine (C-X-C motif) ligand 5 (Cxcl5) [022214] \\ Chemokine (C-C motif) ligand $2(\mathrm{Ccl} 2)$ [31530] \\ Chemokine (C-C motif) ligand $20(\mathrm{Ccl} 20)$ [019233] \\ Chemokine (C-C motif) ligand 5 (Cc15) [031116] \\ Chemokine (C-X3-C motif) ligand 1 (Cx3cl1) [134455] \\ Chemokine (C-C motif) ligand $11(\mathrm{Ccl11})$ [019205] ${ }^{\mathrm{a}}$ \\ Chemokine (C-C motif) ligand $3(\mathrm{Ccl} 3)$ [013025] ${ }^{\mathrm{a}}$ \\ Chemokine (C motif) ligand 1 (Xcl1) [134361] ${ }^{\mathrm{a}}$ \\ Chemokine (C-C motif) receptor 1 (Ccr1) [020542] ${ }^{\mathrm{a}}$
}


Table I. Continued.

\begin{tabular}{|c|c|}
\hline Cascade & Gene [Gene accession no. (NM_)] \\
\hline Complement & $\begin{array}{l}\text { Complement component factor H (Cfh) [130409] } \\
\text { Complement component } 4 \text {, gene } 2 \text { (C4-2) [12805] } \\
\text { Complement component } 6 \text { (C6) [176074] } \\
\text { Complement component } 3 \text { (C3) [016994] }\end{array}$ \\
\hline Adhesion molecule & $\begin{array}{l}\text { Cadherin } 13 \text { (Cdh13) [138889] } \\
\text { Claudin } 3 \text { (Cldn3) [031700] } \\
\text { Claudin } 1 \text { (Cldn1) [031699] } \\
\text { l-gicerin [023983] } \\
\text { Vascular cell adhesion molecule } 1 \text { (Vcam1) [12889] } \\
\text { Selectin, endothelial cell (Sele) [138879] } \\
\text { Junctional adhesion molecule } 3 \text { (Jam3) [1004269] } \\
\text { Neurofascin (Nfasc) [053909] } \\
\text { CD2 antigen (Cd2) [012830] } \\
\text { Junction adhesion molecule 2 (Jam2) [1034004] } \\
\text { Neuron-glia-CAM-related cell adhesion molecule (Nrcam) [013150] } \\
\text { Neurexin } 1 \text { (Nrxn1) [021767] } \\
\text { Intercellular adhesion molecule 2 (Icam2) [1007725] }\end{array}$ \\
\hline Apoptosis & $\begin{array}{l}\text { Bcl-2-related ovarian killer protein (Bok) [017312] } \\
\text { Caspase } 1 \text { (Casp1) [012762] } \\
\text { Bcl2-interacting killer (Bik) [053704] } \\
\text { Tumor necrosis factor receptor superfamily, member } 4 \text { (Tnfrsf4) [013049] } \\
\text { Caspase } 7 \text { (Casp7) [022260] } \\
\text { Protein phosphatase } 2 \text { (formerly 2A), catalytic subunit, } \alpha \text { isoform (Ppp2ca) [017039] } \\
\text { Fragile X mental retardation gene 1, autosomal homolog (Fxr1h) [1012179] } \\
\text { Thioredoxin 1 (Txn1) [053800] } \\
\text { Fas apoptotic inhibitory molecule (Faim) [080895] } \\
\text { CD2 antigen (Cd2) [012830] } \\
\text { v-raf-1 murine leukemia viral oncogene homolog 1 (Raf1) [012639] } \\
\text { Phosphatidylserine receptor (Ptdsr) [1012143] } \\
\text { Myeloid differentiation primary response gene 88 (Myd88) [198130] } \\
\text { FGF receptor activating protein 1 (Frag1) [053895] } \\
\text { Growth arrest specific 6 (Gas6) [057100] } \\
\text { Peroxiredoxin 2 (Prdx2) [017169] } \\
\text { Myelin and lymphocyte protein, T-cell differentiation protein (Mal) [012798] } \\
\text { Tumor necrosis factor (TNF superfamily, member 2) (Tnf) [012675] } \\
\text { p21 protein (cip1) [41275] } \\
\text { Growth arrest and DNA-damage-inducible 45 } \alpha \text { (Gadd45a) [024127] } \\
\text { Fas ligand (TNF superfamily, member 6) (Faslg) [012908] } \\
\text { Cytochrome c, somatic (Cycs) [012839] } \\
\text { Proprotein convertase subtilisin/kexin type 9 (Pcsk9) [199253] } \\
\text { Breast cancer anti-estrogen resistance 1 (Bcar1) [012931] } \\
\text { Bcl2 modifying factor (Bmf) [139258] }\end{array}$ \\
\hline
\end{tabular}

${ }^{\mathrm{a} G e n e}$ whose expression was downregulated.

(MCP-1, CCL2) and RANTES (CCL5) were upregulated in the intima of rat CAs. Complement $\mathrm{C} 3$ and $\mathrm{C} 6$ were also upregulated in the intima. Among adhesion molecules, several genes for family members of claudin and cadherin as well as vascular cell adhesion molecule-1 (VCAM-1) were upregulated in the intima. Apoptosis-related genes upregulated in the intima included a variety of proapoptotic genes such as Bcl-2 family members (Bok, Bik, Bmf), caspases (caspase-1 and -7), Fas ligand and tumor necrosis factor receptor superfamily (TNFRSF), whereas some antiapoptotic genes such as p21 and Fas apoptotic inhibitory molecule (FAIM) were also included. 
Gene expression profile in the media of aneurysmal walls in rats (Table II). Of the 41,012 genes examined, 1344 genes $(3.27 \%)$ were differentially expressed between a normal cerebral artery and a cerebral aneurysm in the media, with 928 genes showing increased expression and 416 genes displaying decreased expression. In addition to MMP-2 and cathepsin Z, cathepsin B, D and Q were upregulated in the media. Tissue inhibitor of MMP (TIMP)-3 was upregulated, and cystatin $\mathrm{C}$, an inhibitor of cysteine cathepsins, was downregulated in the media. Among ROS-related genes, both superoxide-producing genes such as inducible nitric oxide synthase (iNOS, Nos2) and superoxide-eliminating genes such as catalase were upregulated in the media. A variety of growth factors including family members of FGF, TGF and BMP were also upregulated in the media. However, FGF-3, FGF-12 and IL-4 were downregulated in the media. There was a similar expression pattern of chemokines between the intima and media. MCP-1 and RANTES were upregulated also in the media. Contrary to the upregulation in the intima, complement $\mathrm{C} 3$ and $\mathrm{C} 6$ were downregulated in the media. In addition to VCAM-1 and claudins, adhesion molecules between cells and ECM such as laminin, integrin and syndecan were upregulated in the media. Many apoptosis-related genes including caspases, bcl-2 family members and TNFRSF were upregulated in the media.

\section{Discussion}

The distinct features of CAs are inflammatory infiltrate and degenerative changes in vascular walls (11). In the experimentally induced CA model used in the present study, CAs were induced at the cerebral arterial bifurcation by hemodynamic stress and systemic hypertension. Induced CAs share common pathological features with human CAs. Rat CAs show a thinning of the media due to a decrease in the number of medial SMCs (12) and disruption of the internal elastic lamina (13). Some inflammatory cells, especially macrophages, accumulate in the walls of rat CAs (14). Therefore, the results from the gene expression profiling of an experimentally induced CA in rats would provide us with information suggesting the pathophysiology of human CAs. In our experimentally induced CA model of rats, CAs were induced and progressed after aneurysm induction. One month after aneurysm induction, approximately half of the rats showed early aneurysmal change, which was characterized by the disruption of internal elastic lamina without outward bulging of arterial walls. Three months after aneurysm induction, almost all rats displayed advanced aneurysm, which was characterized by the disruption of the internal elastic lamina and the thinning of the media with apparent outward bulging of arterial walls (15). We selected the time period of one month after aneurysm induction in the present study because the pathological changes in the arterial walls rapidly progressed.

Family members of MMPs are thought to play a critical role in the development of various vascular diseases including atherosclerosis $(16,17)$ and aortic abdominal aneurysm (AAA) (18-20) by causing vascular remodeling in arterial walls. We recently demonstrated that macrophage-derived MMP-2 and -9 also participated in CA progression (14). Although macrophages mainly produce MMPs in aneurysmal walls, ECs and SMCs also produce MMPs. In the present study, MMP-2 expression was upregulated in both the intima and media, reflecting upregulated expression of MMP-2 in ECs and SMCs. Notably, TIMP-3, the endogenous inhibitor of MMPs, was also upregulated in the intima. We reported that TIMP-1 and -2 are upregulatedly expressed in SMCs especially in the early stage of CA formation (15). As TIMP-3 has an inhibitory effect on MMP-2 and -9, TIMP-3 may also play a role in $\mathrm{CA}$ formation and progression. Another proteinase inhibitor whose expression was altered with CA formation was cystatin $\mathrm{C}$, an inhibitor for cysteine cathepsins. Cystatin $\mathrm{C}$ expression was downregulated in the media of a rat $\mathrm{CA}$, suggesting the importance of the cathepsin/cystatin $\mathrm{C}$ imbalance in CA formation.

Oxidative stress contributes importantly to the pathophysiology of vascular inflammation. Oxidative stress can be defined as tissue damage occurring secondary to increased production and/or decreased destruction of ROS. Thus, the imbalance between production and destruction of ROS causes increased oxidative stress. In cerebral aneurysmal walls, both ROS-generating genes and antioxidant enzymes were upregulately expressed. We previously reported that iNOS promoted cerebral aneurysm progression $(21,22)$. As the role of oxidative stress has been established in the progression of AAAs (23), it is likely that oxidative stress is increased in cerebral aneurysmal walls and participates in the pathogenesis of CAs.

Increased expression of various inflammatory cytokines and chemokines in cerebral aneurysmal walls strongly suggests that inflammation is deeply involved in the process of CA formation. Some chemoattractants for monocytes including CCL2 (MCP-1) were upregulated both in the intima and in the media. Macrophages accumulate into CA walls and are considered to be a major mediator of the inflammatory reaction in aneurysmal walls $(2,14)$. VCAM-1, which also plays a major role in macrophage recruitment as a cell adhesion molecule for monocytes, was upregulated in aneurysmal walls, supporting the notion that macrophagederived chronic inflammation plays a major role in the process of CA formation.

Some previous reports have revealed complement deposition and activation in human CAs $(2,24)$. The present study showed complex results for complement expression in aneurysmal walls. For example, C3, which triggers the alternative pathway, was upregulated in the intima and downregulated in the media. There may be a different regulation of complement mRNA expression between ECs and SMCs in CAs.

Apoptosis in medial SMCs is one of the prominent features of human CAs $(4,5)$. We previously demonstrated apoptosis of SMCs in experimentally induced rat CAs (25). Many proapoptotic genes such as Bcl-2 family members, Fas ligand and TNFRSF were upregulated both in the intima and in the media, suggesting that complex apoptotic cascades are activated in aneurysmal walls. In addition, IL-1ß, a potent proinflammatory cytokine, was also upregulated in aneurysmal walls in the present study. We previously demonstrated that CA progression was impaired in IL-1ß-deficient mice (26). In IL-1ß-deficient mice, apoptotic cell death in medial SMCs 
Table II. List of genes whose expression changed in the media.

Cascade

Genes [Gene accession no. (NM_)]

Proteinase

ROS

Growth factor
Matrix metallopeptidase 2 (Mmp2) [031054]

Serine (or cysteine) proteinase inhibitor, clade H, member 1 (Serpinh1) [017173]

Serine (or cysteine) peptidase inhibitor, clade B, member 6a (Serpinb6a) [199085]

Cathepsin B (Ctsb) [022597]

Tissue inhibitor of metalloproteinase 3 (Timp3) [12886]

Cathepsin Q (Ctsq), mRNA [139262]

Matrix metallopeptidase 14 (membrane-inserted) (Mmp14) [031056]

Proteasome (prosome, macropain) subunit, ß type 1 (Psmb1) [053590]

Cathepsin Z (Ctsz) [183330]

Synapsin I (Syn1) [019133]

Proteasome (prosome, macropain) subunit, $\beta$ type 4 (Psmb4) [031629]

Serine (or cysteine) peptidase inhibitor, clade A), member 12 (Serpina12) [138825]

Prochymosin (LOC56825), mRNA [020091]

Matrix metalloproteinase 9 (Mmp9) [31055]

Serine (or cysteine) peptidase inhibitor, clade D, member 1 (Serpind1) [024382]

Serine (or cysteine) peptidase inhibitor, clade G, member 1 (Serping1) [199093]

a disintegrin and metallopeptidase domain 6 (Adam6) [138906]

Matrix metallopeptidase 24 (Mmp24) [031757]

Matrix metallopeptidase 3 (Mmp3) [133523]

Cathepsin D (Ctsd) [134334]

Cystatin C [12837] ${ }^{\mathrm{a}}$

Arachidonate 15-lipoxygenase (Alox15) [031010]

Nitric oxide synthase 2, inducible (NOS2) [12611]

Transaldolase 1 (Taldo1) [031811]

Epoxide hydrolase 2, cytoplasmic (Ephx2) [022936]

Peroxiredoxin $2(\operatorname{Prdx} 2)$ [017169]

Growth differentiation factor 15 (Gdf15) [019216]

Pleiotrophin (Ptn) [017066]

Hepatoma-derived growth factor (Hdgf) [053707]

Chemokine (C-X-C motif) ligand 1 (Cxcl1) [030845]

Growth differentiation factor 6 (Gdf6) [1013038]

Fibroblast growth factor 22 (Fgf22) [130751]

Melanoma inhibitory activity 1 (Mia1) [030852]

Hepatocyte growth factor activator (Hgfac) [053320]

Neurotrophic tyrosine kinase, receptor, type 1 (Ntrk1) [021589]

Epidermal growth factor (Egf) [012842]

Bone morphogenetic protein 4 (Bmp4) [012827]

Vascular endothelial growth factor B (Vegfb) [053549]

Bone morphogenetic protein 6 (Bmp6) [013107]

Suppressor of cytokine signaling 2 (Socs2) [058208]

Growth differentiation factor 9 (Gdf9) [021672]

Fibroblast growth factor (acidic) intracellular binding protein (Fibp) [172334]

Myotrophin (Mtpn) [024374]

Neuropilin 1 (Nrp1) [145098]

Transforming growth factor $\beta$ regulated gene 4 (Tbrg4) [1012154]

Fibroblast growth factor 20 (Fgf20) [023961]

Growth differentiation factor 8 (Gdf8) [019151]

Growth differentiation factor 10 (Gdf10) [024375]

Fibroblast growth factor 13 (Fgf13) [053428]

Fibroblast growth factor 21 (Fgf21) [130752]

v-erb-b2 erythroblastic leukemia viral oncogene homolog 2 (Erbb2) [017003]

Bone morphogenetic protein 15 (Bmp15) [021670] 
Table II. Continued.

\begin{tabular}{|c|c|}
\hline Cascade & Genes [Gene accession no. (NM_)] \\
\hline & 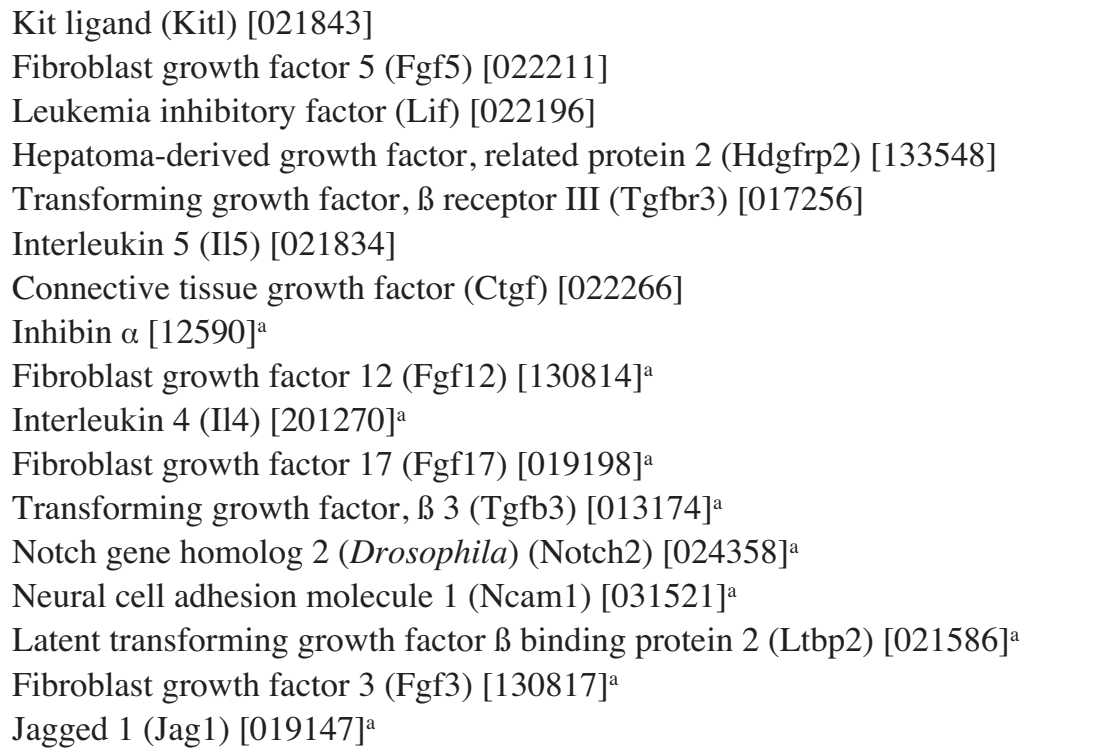 \\
\hline Chemokine & 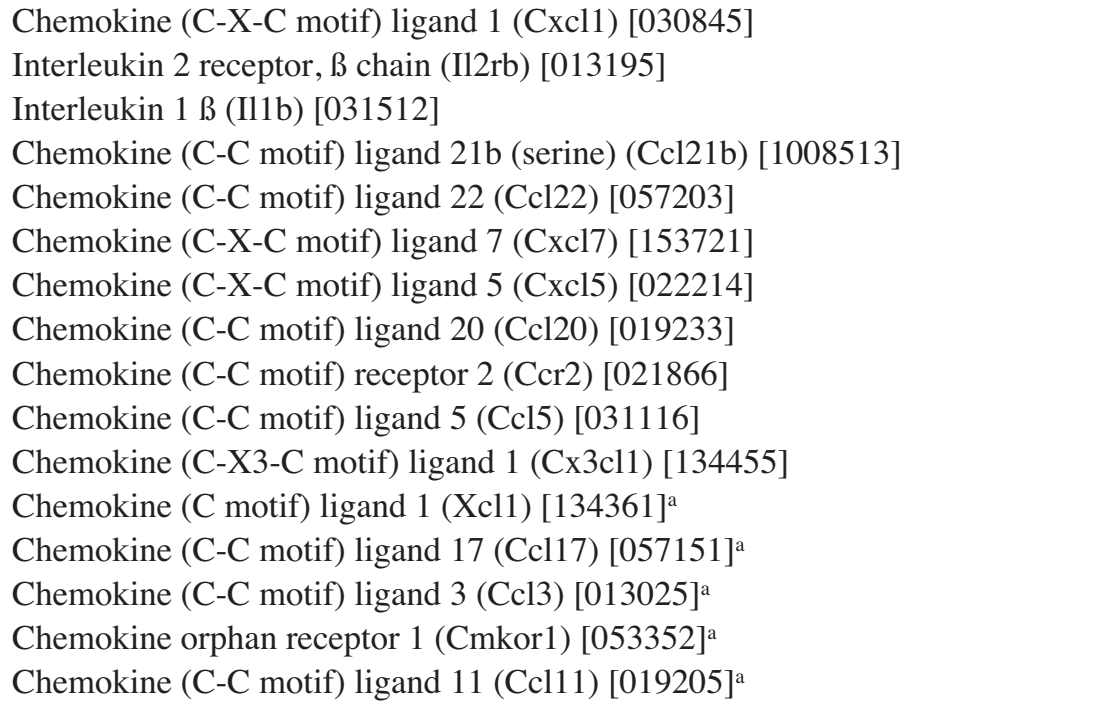 \\
\hline Complement & $\begin{array}{l}\text { Complement component } 1 \text {, q subcomponent, receptor } 1 \text { (C1qr1) [053383] } \\
\text { Complement component 3a receptor } 1 \text { (C3ar1) [032060] } \\
\text { Complement receptor related protein (Crry), transcript variant } 1 \text { [1005330] } \\
\text { Complement component } 6 \text { (C6) [176074] }]^{\mathrm{a}} \\
\text { Complement component } 1 \text {, q subcomponent, B polypeptide (C1qb) [019262] } \\
\text { Complement component } 3(\mathrm{C} 3)[016994]^{\mathrm{a}} \\
\text { Complement component } 4 \mathrm{a}(\mathrm{C} 4 \mathrm{a})[031504]^{\mathrm{a}} \\
\text { Complement component factor } \mathrm{H}(\mathrm{Cfh})[130409]^{\mathrm{a}}\end{array}$ \\
\hline Adhesion molecule & $\begin{array}{l}\text { Laminin, } \beta 2 \text { (Lamb2) [012974] } \\
\text { Neurexin } 2 \text { (Nrxn2) [053846] } \\
\text { Cell adhesion molecule-related/down-regulated by oncogenes (Cdon) [017358] } \\
\text { Cadherin } 15 \text { (Cdh15) [207613] } \\
\text { Junctional adhesion molecule } 3 \text { (Jam3) [1004269] } \\
\text { Cerebral endothelial cell adhesion molecule 1 (Ceecam1) [001011962] } \\
\text { l-gicerin (Mcam) [023983] } \\
\text { CD2 antigen (Cd2) [012830] } \\
\text { Claudin } 14 \text { (Cldn14) [1013429] } \\
\text { Selectin, platelet (Selp) [013114] } \\
\text { Claudin } 5 \text { (Cldn5) [031701] }\end{array}$ \\
\hline
\end{tabular}


Table II. Continued.

Claudin 1 (Cldn1) [031699]

Integrin ß 4 (Itgb4) [013180]

Cell adhesion molecule JCAM (LOC304000) [1006990]

Claudin 3 (Cldn3) [031700]

Acidic (leucine-rich) nuclear phosphoprotein 32 family, member A (Anp32a) [012903]

Myelin-associated glycoprotein (Mag) [017190]

Selectin, endothelial cell (Sele) [138879]

Integrin $\alpha$ M (Itgam) [012711]

Claudin 1 (Cldn1) [031699]

Contactin 3 (Cntn3) [019329]

Syndecan 4 (Sdc4) [012649]

CD4 antigen (Cd4) [012705]

Testicular cell adhesion molecule 1 (Tcam1) [021673]

CD6 antigen (Cd6) [175577]

Intercellular adhesion molecule 1 (Icam1) [012967]

Integrin B 1 (fibronectin receptor B) (Itgb1) [017022]

Vascular cell adhesion molecule 1 (Vcam1) [012889]

Tumor necrosis factor receptor superfamily, member 4 (Tnfrsf4) [013049]

Fas apoptotic inhibitory molecule 2 (Faim2) [144756]

Sequestosome 1 (Sqstm1), transcript variant 2 [181550]

Interleukin 1 (IL1b) [031512]

CD74 antigen [013069]

Presenilin 2 (Psen2) [031087]

Bcl-2-related ovarian killer protein (Bok) [017312]

Cholecystokinin (Cck) [012829]

Squamous cell carcinoma antigen recognized by T-cells 1 (Sart1) [031596]

$\mathrm{R}$ cyclin-dependent kinase 5, regulatory subunit 1 (p35) (Cdk5r1) [053891]

Caspase 6 (Casp6) [031775]

TSC22 domain family 3 (Tsc22d3) [031345]

Caspase 7 (Casp7) [022260]

CD2 antigen (Cd2) [012830]

MAP-kinase activating death domain (Madd) [053585]

Autophagy-related 12 (yeast) (Atg12) [1038495]

Fragile X mental retardation gene 1, autosomal homolog (Fxr1h) [1012179]

Myelin and lymphocyte protein, T-cell differentiation protein (Mal) [012798]

Interleukin-1 receptor-associated kinase 2 (Irak2) [1025422]

v-raf-1 murine leukemia viral oncogene homolog 1 (Raf1) [012639]

p21 protein (cip1) gene [41275]

Bcl2-interacting killer (Bik) [053704]

ras homolog gene family, member A (Rhoa) [057132]

Caspase 12 (Casp12) [130422]

Cyclin-dependent kinase inhibitor 2A (Cdkn2a) [031550]

Peroxiredoxin 2 (Prdx2) [017169]

Cyclin-dependent kinase inhibitor 1A (Cdkn1a), [080782]

Heme oxygenase (decycling) 1 (Hmox 1) [012580]

B-cell translocation gene 1, anti-proliferative (Btg1) [017258]

Fas apoptotic inhibitory molecule (Faim) [080895]

Fas ligand (TNF superfamily, member 6) (Faslg) [012908]

Death associated protein 3 (Dap3) [1011950] 
was also reduced. IL-1ß may be a potent inducer of SMC apoptosis in aneurysmal walls.

In the present study, we described the gene expression profiles in cerebral aneurysmal walls for the first time. Various genes related to inflammation, ECM remodeling and apoptosis were dynamically regulated in cerebral aneurysmal walls. Differences existed in the gene expression profiles between the intima and the media indicating dissimilar roles for ECs and SMCs in CA progression. The findings in the present study provide a novel insight into the pathogenesis of CAs.

\section{Acknowledgements}

This work was supported by a Grant-in-Aid for Scientific Research (no. 17390399) from the Ministry of Education, Science and Culture of Japan.

\section{References}

1. Wiebers DO, Whisnant JP, Huston J III, et al: Unruptured intracranial aneurysms: natural history, clinical outcome, and risks of surgical and endovascular treatment. Lancet 362: 103-110, 2003.

2. Chyatte D, Bruno G, Desai S and Todor DR: Inflammation and intracranial aneurysms. Neurosurgery 45: 1137-1146, 1999.

3. Bruno G, Todor R, Lewis I and Chyatte D: Vascular extracellular matrix remodeling in cerebral aneurysms. J Neurosurg 89: 431-440, 1998

4. Hara A, Yoshimi N and Mori H: Evidence for apoptosis in human intracranial aneurysms. Neurol Res 20: 127-130, 1998.

5. Sakaki T, Kohmura E, Kishiguchi T, Yuguchi T, Yamashita T and Hayakawa T: Loss and apoptosis of smooth muscle cells in intracranial aneurysms. Studies with in situ DNA end labeling and antibody against single-stranded DNA. Acta Neurochir 139: 469-474, 1997.

6. Hashimoto N, Handa H and Hazama F: Experimentally induced cerebral aneurysms in rats. Surg Neurol 10: 3-8, 1978.

7. Morimoto M, Miyamoto S, Mizoguchi A, Kume N, Kita T and Hashimoto N: Mouse model of cerebral aneurysm - Experimental induction by renal hypertension and local hemodynamic changes. Stroke 33: 1911-1915, 2002.

8. Hashimoto N, Kim C, Kikuchi H, Kojima M, Kang Y and Hazama F: Experimental induction of cerebral aneurysms in monkeys. J Neurosurg 67: 903-905, 1987.

9. Hashimoto N, Handa H and Hazama F: Experimentally induced cerebral aneurysms in rats. Part III. Pathology. Surg Neurol 11: 299-304, 1979.

10. Nagata I, Handa H, Hashimoto N and Hazama F: Experimentally induced cerebral aneurysms in rats: Part VI. Hypertension. Surg Neurol 14: 477-479, 1980 .
11. Stehbens WE: Etiology of intracranial berry aneurysms. J Neurosurg 70: 823-831, 1989.

12. Hazama F, Kataoka H, Yamada E, et al: Early changes of experimentally induced cerebral aneurysms in rats. Lightmicroscopic study. Am J Pathol 124: 399-404, 1986.

13. Kim C, Kikuchi H, Hashimoto N, Kojima M, Kang Y and Hazama F: Involvement of internal elastic lamina in development of induced cerebral aneurysms in rats. Stroke 19: 507-511, 1988.

14. Aoki T, Kataoka H, Morimoto M, Nozaki K and Hashimoto N: Macrophage-derived matrix metalloproteinase-2 and -9 promote the progression of cerebral aneurysms in rats. Stroke 38: 162-169, 2007.

15. Aoki T, Kataoka $\mathrm{H}$, Moriwaki $\mathrm{T}$, Nozaki $\mathrm{K}$ and Hashimoto $\mathrm{N}$ : Role of TIMP-1 and TIMP-2 in the progression of cerebral aneurysms. Stroke 38: 2337-2345, 2007

16. Lijnen HR: Metalloproteinases in development and progression of vascular disease. Pathophysiol Haemost Thromb 33: 275-281, 2003.

17. Newby AC: Dual role of matrix metalloproteinases (matrixins) in intimal thickening and atherosclerotic plaque rupture. Physiol Rev 85: 1-31, 2005.

18. Freestone T, Turner RJ, Coady A, Higman DJ, Greenhalgh RM and Powell JT: Inflammation and matrix metalloproteinases in the enlarging abdominal aortic-aneurysm. Arterioscler Thromb Vasc Biol 15: 1145-1151, 1995.

19. Pyo R, Lee JK, Shipley JM, et al: Targeted gene disruption of matrix metalloproteinase-9 (gelatinase B) suppresses development of experimental abdominal aortic aneurysms. J Clin Invest 105: 1641-1649, 2000.

20. Thompson RW, Holmes DR, Mertens RA, et al: Production and localization of 92-kilodalton gelatinase in abdominal aorticaneurysms - an elastolytic metalloproteinase expressed by aneurysm-infiltrating macrophages. J Clin Invest 96: 318-326, 1995.

21. Fukuda S, Hashimoto N, Naritomi H, et al: Prevention of rat cerebral aneurysm formation by inhibition of nitric oxide synthase. Circulation 101: 2532-2538, 2000.

22. Sadamasa N, Nozaki K and Hashimoto N: Disruption of gene for inducible nitric oxide synthase reduces progression of cerebral aneurysms. Stroke 34: 2980-2984, 2003.

23. McCormick ML, Gavrila D and Weintraub NL: Role of oxidative stress in the pathogenesis of abdominal aortic aneurysms. Arterioscler Thromb Vasc Biol 27: 461-469, 2007.

24. Tulamo R, Frosen J, Junnikkala S, et al: Complement activation associates with saccular cerebral artery aneurysm wall degeneration and rupture. Neurosurgery 59: 1069-1076, 2006.

25. Kondo S, Hashimoto N, Kikuchi H, Hazama F, Nagata I and Kataoka H: Apoptosis of medial smooth muscle cells in the development of saccular cerebral aneurysms in rats. Stroke 29: $181-188,1998$

26. Moriwaki T, Takagi Y, Sadamasa N, Aoki T, Nozaki K and Hashimoto N: Impaired progression of cerebral aneurysms in interleukin-1beta-deficient mice. Stroke 37: 900-905, 2006. 